\title{
Indefinición del Concepto de Adulto Mayor Laboralmente Activo en Colombia
}

\author{
Lack of Definición of the Concept of Labor Older Adults in Colombia \\ ${ }^{a}$ Nataly Andrea García Mejía ${ }^{49}$ \\ a*ngarciam@unicartagena.edu.co “Semillero Políticas Públicas Participación y Desarrollo, Programa de Derecho, Universidad de Cartagena. \\ Cartagena, Colombia.
}

Forma de citar: N.A. García-Mejía "Indefinición del Concepto de Adulto Mayor Laboralmente Activo en Colombia”, Rev. Saberes, Vol. 01, No. XX, pp. $63-67,2020$.

Recibido: 24/03/2020 Evaluación: 28/05/2020 Aceptado: 30/06/2020 DOI: https://doi.org/10.25213/1794-4384/1302.0008

\section{Resumen}

De conformidad con el estudio "Misión Colombia Envejece”, a partir del año 2020 el país experimentará un crecimiento de los adultos mayores en los próximos años, pronosticándose para el año 2050 un aumento del $10.8 \%$ al $23 \%$, equivalente a poco más de 14 millones de personas. Actualmente, la mayoría de quienes conforman este grupo se ven forzados a mantenerse laboralmente activos para garantizar su subsistencia. Con ello, se crea la necesidad de implementar políticas públicas dirigidas a este sector poblacional. Mediante el presente escrito, se determinarán los criterios utilizados para identificar quiénes pueden ser considerados como adultos mayores en el derecho laboral colombiano.

Como conclusión, se observa la prevalencia del factor edad al momento de estimar la vejez laboral del sujeto, pero se omiten circunstancias visibilizadas tanto por el Derecho Internacional del Trabajo como por la jurisprudencia nacional y la sociedad colombiana. Lo anterior devenga en una falta de precisión sobre los criterios a tener en cuenta para identificar a esta población.

\section{Palabras Clave}

Adulto mayor, indefinición, políticas públicas, edad, derecho laboral.

\begin{abstract}
According to the "Mission Colombia Aging" study, from 2020 the country will experience a growth of older adults in the coming years, with an increase of $10.8 \%$ to $23 \%$ being predicted by 2050 , equivalent to just over 14 million of people. Currently, most of those who make up this group are forced to remain active in work to ensure their livelihood. This creates the need to implement public policies aimed at this population sector. Through this document, the criteria used to identify who can be considered as older adults in Colombian labor law will be determined. In conclusion, the prevalence of the age factor is observed when estimating the subject's old age, but circumstances visible both by International Labor Law and by national jurisprudence and Colombian society are omitted. The above results in a lack of precision about the criteria to be taken into account to identify this population.
\end{abstract}

\section{Keywords}

Older adults, vagueness, public policies, age, Labor law.

\section{Introducción}

Dado el engrosamiento de la población mayor dentro de los próximos años, en Colombia se ha convertido en asunto de interés el desarrollo de políticas públicas dirigidas a la tutela de los derechos fundamentales de quienes conforman

\footnotetext{
${ }^{49}$ Autor para correspondencia: correo electrónico ngarciam@unicartagena.edu.co (C) 2020 Fundación Universitaria Antonio de Arévalo - UNITECNAR. Este es un artículo bajo la licencia CC BY-NC-ND (http://creativecommons.org/licenses/by nc-nd/4.0/)
} 
este sector poblacional, convirtiéndose no solo en algo necesario dada las expectativas sino esencial para estas personas consideradas de especial protección constitucional.

Es aquí donde el derecho laboral juega un papel importante, por cuanto uno de sus objetivos es proporcionar a la persona las garantías para su autorrealización, así como su propio sostenimiento (Mendoza Martelo, 2010). Sin embargo, al momento de esclarecer quiénes pertenecen a la categoría de "adulto mayor", existen discrepancias en la ley, la jurisprudencia y percepción de la población sobre los factores determinantes.

El escrito presentado a continuación explica, de forma concisa, la problemática existente dentro del ordenamiento jurídico al momento de identificar qué parte de la población corresponde a adultos mayores desde la óptica del derecho laboral; más específicamente, ¿bajo qué criterios se puede considerar a una persona un adulto mayor en el derecho laboral colombiano?

Para ello, se expondrá la visualización existente en torno al adulto mayor dentro de las leyes y la jurisprudencia nacional; posteriormente, se definirá su situación en el campo laboral y finalmente se identificarán tanto los criterios implementados en el derecho laboral colombiano como las inconsistencias presentes en los factores hallados para determinar cuándo una persona laboralmente activa es un adulto mayor; para lo cual se utilizará una metodología de carácter exploratorio, a través de la revisión de artículos investigativos, leyes, jurisprudencia y artículos periodísticos en torno al problema planteado.

\section{Resultados y Discusión}

De acuerdo a Arango, Ruiz y Fundación Saldarriaga Concha (s.f.), la población mayor en Colombia "ha perdido poderes y derechos que lo han marginado de la sociedad creando una serie de estereotipos negativos, de enfermedad, incapacidad de producción y de asumir tareas y ejercer funciones".

\footnotetext{
50 Yánez, Maldonado y Del Risco (2016) resaltan que para las políticas nacionales "no considera[n], o por lo menos no como un eje central de acción, el acceso del adulto mayor a instrucción
}

Al parecer, las políticas públicas dirigidas al adulto mayor en el país se impregnan de esta concepción. Éstas, en su mayoría, se inclinan hacia lo humanitario $^{50}$ - entrega de subsidios y prestaciones - por cuanto se visualiza al adulto mayor como una persona improductiva que, por sus condiciones físicas, está incapacitada para realizar un trabajo de cualquier índole. $\mathrm{Ni}$ la jurisprudencia de la Corte Constitucional, caracterizada por una línea de pensamiento vanguardista, se ha librado de esta generalización: Nuestra Constitución Nacional ha considerado a la persona humana y su dignidad como el presupuesto esencial del nuevo Estado Social de Derecho, y es por esto que ha procurado entre otras cosas, prestar una especial protección a aquellos individuos que se encuentren en situaciones de desventaja, dadas sus condiciones físicas y mentales, frente a los demás. Es así como en la Carta Política de 1991 se señala a las personas de la tercera edad, como uno de los sectores de la población que requieren una asistencia profunda y efectiva del Estado, la sociedad y la familia. Los ancianos son individuos que se encuentran limitados e incluso imposibilitados para adquirir un sustento que les permita vivir dignamente, ya que su capacidad laboral se encuentra prácticamente agotada (1998).

(...) no se puede desconocer los constantes inconvenientes que tienen que afrontar las personas de edad avanzada cuyas condiciones fisicas: (i) les impiden trabajar, (ii) les ocasiona restricciones originadas en las prohibiciones legales que hacen obligatorio el retiro forzoso de su trabajo al arribar a cierta edad, y en consecuencia, (iii) los inhabilita para poder proveerse sus propios gastos (2017). (Cursiva propia)

La realidad, paradójicamente, resulta ser distinta. De acuerdo a Farné y Rodríguez (2014), el adulto mayor se convierte en parte esencial de la economía del hogar, muchas veces encargándose del cuidado y alimentación de otras personas. Además, dado que el 75\% no goza de una pensión y el $47 \%$ posee ingresos personales bajos, muchos se ven obligados a mantenerse laboralmente activos como independientes en actividades

permanente, ya sea formal o informal". Dicha desactualización de conocimientos "se suma a la renuencia por parte del sector productivo en invertir en la formación de personas mayores".

Página | 64 
mayoritariamente informales. En estos trabajos usualmente son objeto de abusos, teniendo que lidiar "con bajos salarios y trato discriminatorio" (Centro de Documentación Judicial y Consejo Superior de la Judicatura, s.f.).

Así las cosas, algo primordial sería la creación de mecanismos de protección encaminados a los adultos mayores laboralmente activos. Sin embargo, dentro de nuestro ordenamiento jurídico hallamos uno de los primeros impedimentos: definir cuándo una persona es, en materia laboral, un adulto mayor.

Una de las primeras disposiciones normativas que señala la entrada a la vejez laboral es el artículo 33 de la Ley 100 de 1993, el cual enuncia los requerimientos para acceder a la pensión de vejez, prestación económica otorgada al trabajador en virtud a "la prestación del servicio durante un número determinado de años, con la concurrencia del factor edad" (Corte Suprema de Justicia, 1958; citado en Corte Constitucional, 1998), señalando que se otorgará a las mujeres que hayan cumplido 57 años y a los hombres que hayan cumplido los 62 años.

En principio, el criterio de edad inmerso en la Ley 100 para determinar la "vejez laboral" por sí solo aparenta ser razonable. Sin embargo, viene siendo necesario complementarlo con otro factor que la normatividad internacional del trabajo ha traído a colación $^{51}$ : la dificultad del sujeto para la obtención de un puesto de trabajo.

Así las cosas, considera la autora, que podrían englobarse dos nuevos grupos que cambiarían el margen dispuesto por la Ley 100 y sobre los cuales la jurisprudencia nacional en algún momento hizo mención acerca de sus dificultades para el acceso al empleo.

Un primer grupo lo conformaría todo sujeto que, de acuerdo con la Corte Constitucional, esté "próximo a pensionarse al cual le falten tres (3) o menos años para reunir los requisitos de edad y tiempo de servicio o semanas de cotización para obtener el disfrute de la pensión de jubilación o vejez" (2009; citado en Corte constitucional,

\footnotetext{
51 La norma en cuestión es la Recomendación 162 de la Organización Internacional del Trabajo (OIT). En este caso, a los adultos mayores se les denomina trabajadores de edad y se les define como "todos los trabajadores que, por el avance de su edad, están expuestos a encontrar dificultades en materia de empleo y ocupación".

Es importante aclarar que las Recomendaciones de la OIT no son vinculantes dentro del ordenamiento jurídico colombiano, pero sí son fuente de inspiración para crear y modificar la normatividad interna.
}

2016), los cuales se les ha denominado prepensionados. El mismo tribunal constitucional ha señalado que el prepensionado "debido a la edad en que se encuentra (...) puede conllevar a que sea difícil conseguir un nuevo empleo y por ende satisfacer las necesidades básicas de un hogar" (Corte Constitucional, 2018), razón por la cual se les garantiza la protección a sus garantías fundamentales.

En este sentido, el umbral de edad para definir la "vejez laboral" se extendería - conforme a la definición de la Corte - a los 54 años para las mujeres y 59 años para los hombres, lo cual implica una disminución al rango de edad contenido en la Ley 100 de 1993.

El segundo grupo lo conformarían los adultos con edad igual o superior a los 40 años. Como se observa en acápites anteriores, la edad se convierte en un criterio excluyente que ha creado presunciones sobre quién es apto para realizar cualquier tipo de actividad. No por nada la población colombiana percibe dificultades para acceder a un empleo formal a partir de los 40 años, sintiéndose discriminados por las políticas de edad manejadas por las empresas. (Portafolio, 2016; Dinero, 2016) ${ }^{52}$.

Sobre el asunto, la Corte Constitucional trae a colación:

Tal como se ha recordado recientemente, "[p]roducto de la conquista del tiempo, la vejez será cada vez menos sinónimo de muerte: muerte biológica vital. La muerte social es la muerte que acompaña a la vejez en la sociedad y en la cultura occidental moderna. Es la exclusión y discriminación por razón de la edad. Cuando se observa el mercado laboral esto se ve claramente. Por el motivo señalado, algunas personas han llegado a proponer un cambio en la definición de trabajadorla mayor que rompa con la noción de proximidad a la edad de jubilación y que se base en una concepción de empleabilidad en relación con el mercado de trabajo y no de marginación o discriminación. Curiosamente después de los 40 años trabajadores/as ya son considerados(as)

\footnotetext{
${ }^{52} \mathrm{Si}$ bien las personas mayores de 40 años poseen la experiencia requerida para el cargo al cual aspiran, algunas de las dificultades que los empleadores observan al momento de contratar son "el poder de adaptabilidad a los cambios, los problemas de salud y las dificultades en el manejo de la tecnología". (Dinero, 2016)
} 
viejos(as)" (Osorio, 2005; citado en Corte Constitucional, 2008).

\section{Conclusiones}

A modo de conclusión, al estudiar la normatividad en torno al adulto mayor, se observa que la edad del trabajador es el factor principal utilizado para su identificación. No obstante, si se suma lo expuesto por el Derecho Laboral Internacional, la Corte constitucional y los colombianos partícipes del mercado laboral nacional, se incluyen nuevos sectores poblacionales, originando una falta de precisión frente a quiénes pueden considerarse de este grupo cuando se hace referencia a aquellos laboralmente activos, inclusive apartándose de la realidad vivida por los afectados.

Se evidencia la necesidad de replantear la conceptualización actual de la vejez para efectos laborales: si debe seguir determinándose tomando como referente la edad - por las condiciones físicas del trabajador o si, por el contrario, debe visualizarse a partir de la percepción de rentabilidad que tiene aquel dentro del mercado laboral, de modo que las políticas públicas que se propongan acierten en el público objetivo y su ejecución resulte efectiva a los problemas que ellos aquejen.

\section{Referencias Bibliográficas}

Arango, V. E., Ruiz, I. C. y Fundación Saldarriaga Concha (s.f.). DIAGNÓSTICO DE LOS ADULTOS MAYORES DE COLOMBIA. http://www.sdp.gov.co/sites/default/files/diag_ad ul_mayor.pdf

Centro de Documentación Judicial y Consejo Superior de la Judicatura. (s.f.). La tercera edad en Sujetos de Especial Protección en la Constitución Política.

http://www.unilibrebaq.edu.co/unilibrebaq/pdhulb q/html/capitulo3.html

Congreso de la República de Colombia. (23 de diciembre de 1993) Ley 100 de 1993. Por la cual se crea el sistema de seguridad social integral y se dictan otras disposiciones. Diario Oficial No. 41.148 .

http://www.secretariasenado.gov.co/senado/based oc/ley 0100 1993.html
Corte constitucional. (1998, 04 de mayo). Sentencia C - 177/98. (Dr. Alejandro Martínez Caballero, $\quad$ M.P.). https://www.corteconstitucional.gov.co/RELATO RIA/1998/C-177-98.htm

Corte constitucional. (1998, 30 de abril). Sentencia $\mathrm{T}$ - 169/98. (Dr. Fabio Morón Díaz, M.P.). https://www.corteconstitucional.gov.co/relatoria/1 998/T-169-98.htm

Corte constitucional. (2008, 02 de diciembre). Sentencia T - 1178/08. (Humberto Antonio Sierra Porto, M.P.) https://www.corteconstitucional.gov.co/relatoria/2 $\underline{008 / \mathrm{T}-1178-08 . h t m}$

Corte constitucional. (2016, 06 de julio). Sentencia $\mathrm{T}$ - 357/16. (Jorge Iván Palacio, M.P.). https://www.corteconstitucional.gov.co/relatoria/2 016/t-357-16.htm

Corte constitucional. (2017, 26 de abril). Sentencia T - 252/17 (Iván Humberto Escrucería Mayolo, M.P.).

https://www.corteconstitucional.gov.co/relatoria/2 017/T-252-17.htm

Corte constitucional. (2018, 09 de agosto). Sentencia T - 325/18. (José Fernando Reyes Cuartas, M.P.). https://www.corteconstitucional.gov.co/relatoria/2 018/T-325-18.htm

Dinero. (2016, 7 de junio). ¿Existen oportunidades laborales para los mayores de 40 años en Colombia?

Dinero.

https://www.dinero.com/economia/articulo/situaci on-laboral-de-personas-mayores-de-40-anos-encolombia/225362

Farné, S. y Rodríguez Guerrero, D.A. (2014, noviembre). PARTICIPACIÓN DE LOS ADULTOS MAYORES EN LAS ECONOMÍAS DE MERCADO Y DEL HOGAR EN COLOMBIA. Observatorio del Mercado de Trabajo y la Seguridad Social - Cuaderno de Trabajo, 16. https://www.uexternado.edu.co/wpcontent/uploads/2017/01/Cuaderno-16.pdf 
Mendoza Martelo, G.E. (2010, septiembre) La jurisprudencia de la Corte Constitucional frente a la creación del trabajo formal. Trabajo presentado en $6^{\circ}$ Encuentro de la Jurisdicción Constitucional de la Corte Constitucional. http://www.corteconstitucional.gov.co/viencuentr o/conferencia $\% 20$ gemm.php.

Organización Internacional del Trabajo. (1980, 23 de junio). Recomendación 162. Recomendación sobre los trabajadores de edad. https://www.ilo.org/dyn/normlex/es/f?p=NORML EXPUB:12100:0::NO::P12100 ILO CODE:R16 $\underline{2}$

Portafolio. (2016, 07 de septiembre). Nos estamos volviendo viejos y quedando sin mano de obra. Portafolio.

https://www.portafolio.co/economia/empleo/cuales-la-edad-mas-dificil-para-conseguir-trabajo-encolombia-500141

Yánez Contreras, M. A., Maldonado Pedroza, C. D. y Del Risco Serje, K. P. (2016) Participación laboral de la población de 60 años de edad o más en Colombia. Economía del Caribe. (17) 39 - 63. http://rcientificas.uninorte.edu.co/index.php/econ omia/article/view/8004/html 314 